\title{
The role of pharmacokinetic and pharmacokinetic/pharmacodynamic modeling in drug discovery and development
}

\author{
"In silico and computational modeling, in conjunction with early absorption, distribution, metabolism and \\ excretion/pharmacokinetic screening and pharmacokinetic/pharmacodyanmic modeling and simulation, will play an increasingly \\ important role in optimizing drug discovery and development in the future."
}

The pharmacokinetic (PK) behavior of a drug is a critical determinant of both its efficacy and safety. Thus, in recent years we have seen a continuing focus on the development of earlier in silico, in vitro and in vivo screening approaches to improve predictivity of human PK behavior. We have seen an increasing plethora of predictive structure-based in silico models, high-throughput in vitro assays for screening absorption, distribution, metabolism and excretion (ADME) properties and earlier PK and $\mathrm{PK} /$ pharmacodynamic (PD) modeling and simulation. These approaches are allowing a more mechanistic basis for translating and predicting a drug's PK/PD behavior in humans. This article discusses some of the approaches being increasingly applied in drug discovery to assist medicinal chemists in the design of molecules with improved $\mathrm{PK}$ and PK/PD properties.

\section{In silico computational modeling of ADME/PK properties}

In recent years we have seen an increasing interest and development of software and computational models for the prediction of ADME/PK properties. While it is not the intent of this article to provide a comprehensive review of the commercially available in silico software for the prediction of ADME/PK properties, some mention is warranted. Several excellent reviews covering ADME/PK software and databases have appeared in recent years [1-3]. These reviews detail some of the ADME properties being modeled by in silico approaches, including solubility, $\mathrm{pKa}$ and metabolism, as well as available metabolism databases. In addition, there are now numerous software packages and statistical tools to support the generation of molecular descriptors and fragmentation patterns to assist in the development of both local and global in silico models from available literature publications and internal company data. Commercial modeling software is also available for the modeling and simulation of clinical trial design and outcome. There continues to be a pressing need to improve the robustness, utility and accuracy of the in silico models for ADME/PK prediction, as in silico and computational modeling, in conjunction with early ADME/PK screening, will continue to play an increasingly important role in optimizing drug discovery and development in future.

\section{Prediction of in vivo clearance from in vitro metabolism data}

Clearance (CL) is one of the most important PK parameters and is defined as the volume of body fluid (e.g., plasma) from which a drug is removed by biotransformation and/or excretion, per unit of time. CL largely governs exposure and the fate of a drug in the body. The prediction of human $\mathrm{CL}$ is critical in drug discovery. High-throughput determination of in vitro metabolic stability and metabolism, typically with liver preparation from preclinical species and humans, has added significant value for medicinal chemists in understanding structure-activity relationships (SAR) for metabolic liability and in expediting drugdiscovery progress. It has been suggested that the determination of intrinsic CL, based on the data obtained from in vitro metabolism assays, may provide an assessment of in vivo CL $[4,5]$. However, the robustness, accuracy and precision of using metabolic stability data to predict in vivo CL appears questionable [6,7]. We have recently examined the relationship between in vitro and in vivo CL for a large group of structurally diverse compounds [8]. In vitro intrinsic CL was derived from metabolic stability in mouse hepatic microsomes, S9 fraction and hepatocytes. The

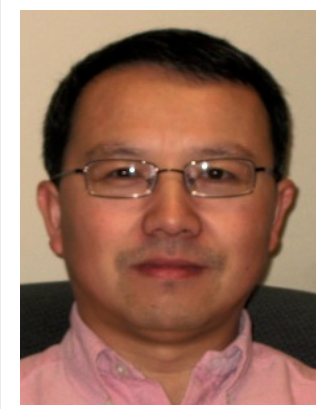

\section{Xiang-Qing Yu}

Drug Metabolism,

Pharmacokinetics, Toxicology

and Pathology, Lexicon

Pharmaceuticals, Inc.,

The Woodlands, TX 7738I, USA

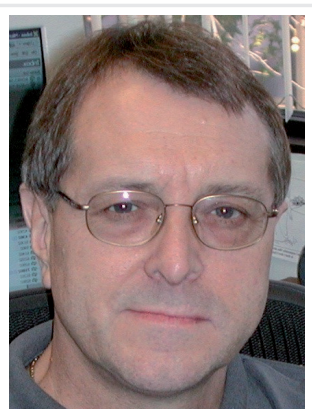

Alan GE Wilson

Author for correspondence: Drug Metabolism,

Pharmacokinetics, Toxicology and Pathology, Lexicon Pharmaceuticals, Inc.,

The Woodlands, TX 7738I, USA

Tel.: + I 28I 8633952

Fax: + I 28I 8633564

E-mail: awilson@lexpharma.com 
predicted hepatic clearance was then compared with the observed in vivo CL in the mouse. Only $45 \%$ of the compounds showed good agreement between the observed in vivo CL and the predicted CL. The incorporation of plasma protein and/or microsome binding into the prediction did not improve the in vitro and in vivo correlation (IVIVC). Many reasons may account for this discrepancy, such as extrahepatic clearance, physicochemical properties, tissue uptake and transportermediated CL. For example, we have recently observed that, for a structurally related group of molecules, in vivo CL increased as polar surface area increased (FIGURE I). Soars et al. reported that the conventional in vitro metabolism assay underpredicted in vivo CL of $20 \%$ of the compounds tested [9], which is consistent with our finding [8]. When incorporating hepatic transporter-mediated uptake into the prediction, IVIVC for those underpredicted compounds was significantly improved [9]. It appears that in vitro metabolism data may help rank compounds and develop SAR, but should be used with caution when trying to predict in vivo CL. To improve in vivo prediction, a more integrated approach such as physiologically based pharmacokinetic (PBPK) modeling may be needed.

\section{PBPK modeling \& simulation}

The early prediction of human PK is playing an increasingly important role in drug discovery. Allometric scaling, an empirical approach, has long been used for interspecies scaling of some PK properties [10]. However, the precision of

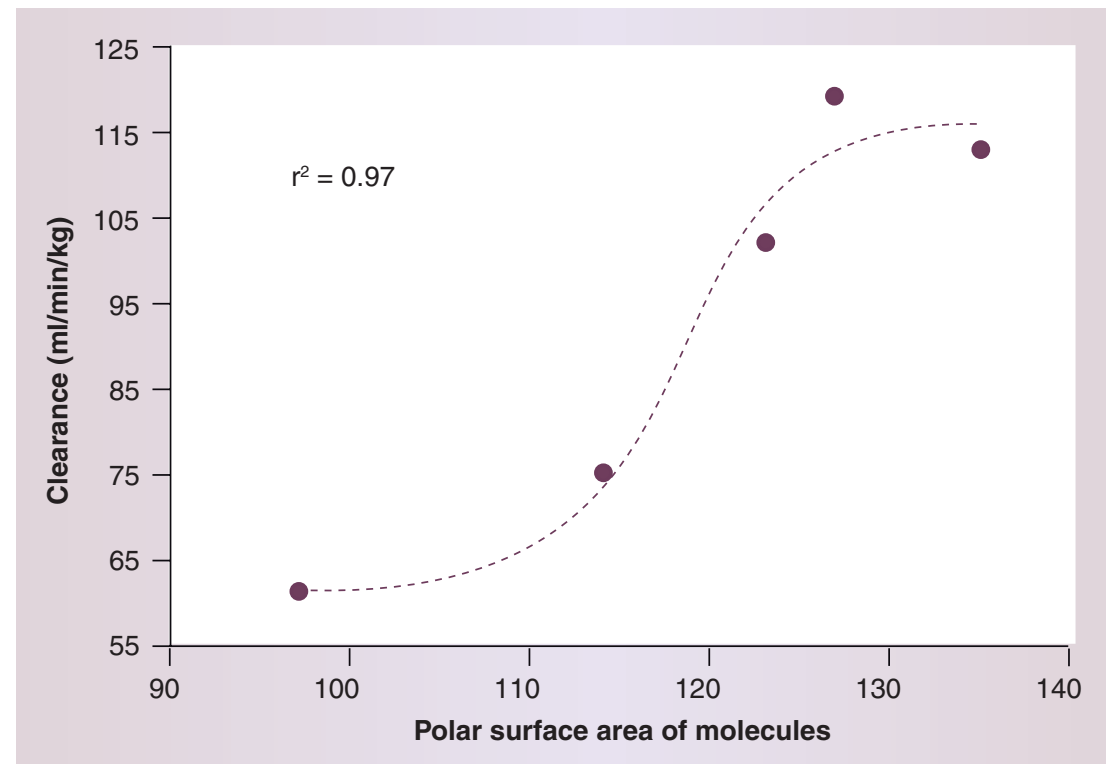

Figure 1. Plasma clearance versus polar surface area. allometric scaling is limited by the uncertainty in the extrapolation, due to the lack of a clear mechanistic basis [11]. By contrast, PBPK modeling is a mechanistically based approach that has been successfully used for simulating the ADME profile of a compound by integrating compound physicochemical properties and in vitro ADME data. Unlike conventional or classical PK models, PBPK models anatomically and physiologically represent the body by incorporating parameters corresponding to actual blood-flow rates, tissue and organ volumes and activities of drug transporters/disposition enzymes [12]. PBPK models have two classes of input parameters: physiological parameters (e.g., organ volume and blood flow) and chemical specific parameters (e.g., molecular weight, solubility, particle size, $\mathrm{pKa}$, LogP, permeability, metabolic stability, Ki and plasma protein binding). Physiological parameters are species dependent and available in the literature [13-15] . Chemical-specific parameters can be readily determined in vitro using available techniques. By inputting these parameters into an appropriate PBPK model, the in vivo $\mathrm{PK}$ profile of a compound can be simulated for different species, including humans. Numerous commercial PBPK software tools are available to facilitate ADME/PK prediction, including GastroPlus ${ }^{\mathrm{TM}}$, SIMCYP ${ }^{\mathrm{TM}}$, acslXtreme, PK-SIM ${ }^{\circledR}$, CloePK ${ }^{\mathrm{TM}}$, IDEA and Madonna $^{\mathrm{TM}}[12,16]$. Some of the software tools simulate particular PK-relevant processes such as oral absorption and metabolism drug-drug interactions. Drug-drug interaction is of significant concern to the pharmaceutical industry, often leading to discontinuation of drug development. Early prediction of drug-drug interaction potential can significantly facilitate candidate selection and assist clinical trial design. The availability of software for the prediction of drug-drug interaction is a continuing area of focus in PK modeling. In addition to small molecules, the application of PBPK was recently expanded to macromolecules, such as monoclonal antibodies [17].

\section{Application of microdose strategy to early drug development}

The concept of a Phase 0 or microdose study was recently introduced as a possibility for accelerating the clinical PK of a new molecular entity [18]. Unlike traditional Phase Ia PK studies, microdose studies are conducted at a very low, subtherapeutic dose $(<1 \%$ the efficacious dose with the maximal dose $\leq 100 \mu \mathrm{g}$ ) [19]. Owing to the low dose, fewer preclinical studies are required to initiate a clinical microdose study [20]. This has 
the potential advantage of shortening the period of obtaining human PK data [20]. In addition, it is finding application in the early selection of a clinical candidate when several potential candidates exist and are difficult to differentiate with preclinical data. However, it is important to recognize that microdosing does have its limitations. For instance, it has not yet been demonstrated that it can significantly cut the time to final regulatory approval. It also has a limitation in that the compound may not be pharmacokinetically linear from a microdose to a therapeutic dose.

\section{PK/PD modeling \& simulation in drug discovery}

The purpose of PK is to investigate what the body does to a drug and PK modeling quantitatively or mathematically describes the processes of drug ADME. On the other hand, PD is the study of what a drug does to the body. PK/PD modeling defines a mathematical relationship between exposure and PD effect and determines how much exposure is needed, and for how long, to exert the effect. The goal of PK/PD modeling is to use the model to predict the exposure, PD effects under various experimental and therapeutic conditions and to ultimately maximize the chance of success of drug candidates. The early development of PK/PD models is aiding both the discovery process and also the translation of preclinical data into the clinic. PK/PD modeling in discovery will continue to receive increasing focus in the future and will be expected to make a significant impact on drug discovery and development.

\section{PK/PD model classification}

Based on the principals of model development, $\mathrm{PK} / \mathrm{PD}$ models can be classified as empirical or mechanistic models [21]. Empirical models emphasize phenomenological relationships between exposure and effect, without regard to the underlying mechanistic underpinnings [21]. There are many empirical models available, which include direct-link models, spline-function models, logistic regression models and circadian models [22]. Circadian models have been commonly used to describe daily fluctuations in blood pressure, serum glucose, QTc interval, hormone concentrations, temperature and circulating white cells [23-27]. Owing to the limited understanding of the pathophysiology of many human diseases, another important utility of empirical models is to describe disease progression (e.g., tumor growth and Alzheimer's disease) [28,29]. For example, in xenograft tumor models, tumors initially grow in an exponential manner followed by a linear fashion [29]. Simeoni et al. developed a unique empirical model that utilizes tumor size as a trigger to change tumor growth from the initial exponential phase to a linear phase [29]. Overall, empirical models still remain as a useful and practical approach in the current drugdiscovery paradigm. Conversely, the mechanistic approach is physiologically based [30]. The development of mechanistic models usually relies on data of pharmacologically/clinically relevant biomarker response. Mechanistic models may provide a better prediction across species, populations and dosing regimens than empirical models [30]. Mechanistic models are being increasingly applied to drug discovery and development [31-34]. Animal studies are particularly important in exploring and developing mechanistically based PK/PD models for drug discovery and provide a valuable tool for translation into the clinic. For instance, 2-acetyl4(5)-tetrahydroxybutyl imidazole (THI) reduces peripheral lymphocytes by increasing lymphoid sphingosine 1-phosphate (S1P) levels through inhibition of S1P lyase. We have recently developed a mechanistically based model that consists of three sub-models: an indirect-II model, a four-step transduction model and a direct-link PD model. The integrated model quantitatively describes how THI could increase splenic S1P level by inhibiting S1P lyase subsequently with a reduction of peripheral lymphocyte counts in rats [31]. This PK-biomarker-PD model was able to predict the PD response at different dose level [31]. Similarly, Beier et al. reported a semimechanistic model (an indirect-II PD model incorporating a competitive interaction model), which successfully described antinorceptive effects following dosing of analgesics to rats [32].

\section{- When should PK/PD modeling \&} simulation be applied to drug discovery?

Pharmacokinetic/pharmacodynamic modeling and simulation can add value to almost any stage of drug discovery. The activity of PK/PD modeling and simulation can be sequentially divided into four levels (TABLE I). In level 0 , the in vivo pharmacology response is solely interpreted by dose-response data. At level 1, PK/PD analysis attempts to correlate PK data with PD data. This simple analysis can sometimes provide a good insight into the relationship between exposure and response. For example, a simple plot of plasma exposure and in vivo PD response of one of our discovery compounds suggests that the efficacious 
Table 1. The activity of pharmacokinetic/pharmacodynamic modeling and simulation.

\begin{tabular}{|c|c|}
\hline Level & PK/PD modeling activity \\
\hline 0 & $\begin{array}{l}\text { In vivo PD response is solely addressed by dose response without using } \\
\text { PK data. }\end{array}$ \\
\hline 1 & $\begin{array}{l}\text { Include PK data into PD and safety studies (to verify PD responses } \\
\text { associated with target/biomarker modulation). }\end{array}$ \\
\hline 2 & $\begin{array}{l}\text { Develop PK/PD model to assign compound and biological system related } \\
\text { parameters (i.e., } \mathrm{EC}_{50^{\prime}} \mathrm{E}_{\text {max }^{\prime}} \mathrm{K}_{\text {eo }}, \mathrm{K}_{\text {in }} \text { and } \mathrm{K}_{\text {out }} \text { ). }\end{array}$ \\
\hline 3 & $\begin{array}{l}\text { Utilize PK/PD models to assist dose-regimen optimization, assist species } \\
\text { translation activity including humans and define therapeutic window. }\end{array}$ \\
\hline
\end{tabular}

dose level may be a dose that can bring the ratio of the average of the unbound plasma exposure/ $\mathrm{IC}_{50}$ to greater than 30 (Figure 2A). The hypothesis was later confirmed by testing an additional eight compounds (FIgure 2B). This principal was thereafter applied to the selection of compounds for testing and in guiding dosing regimens for
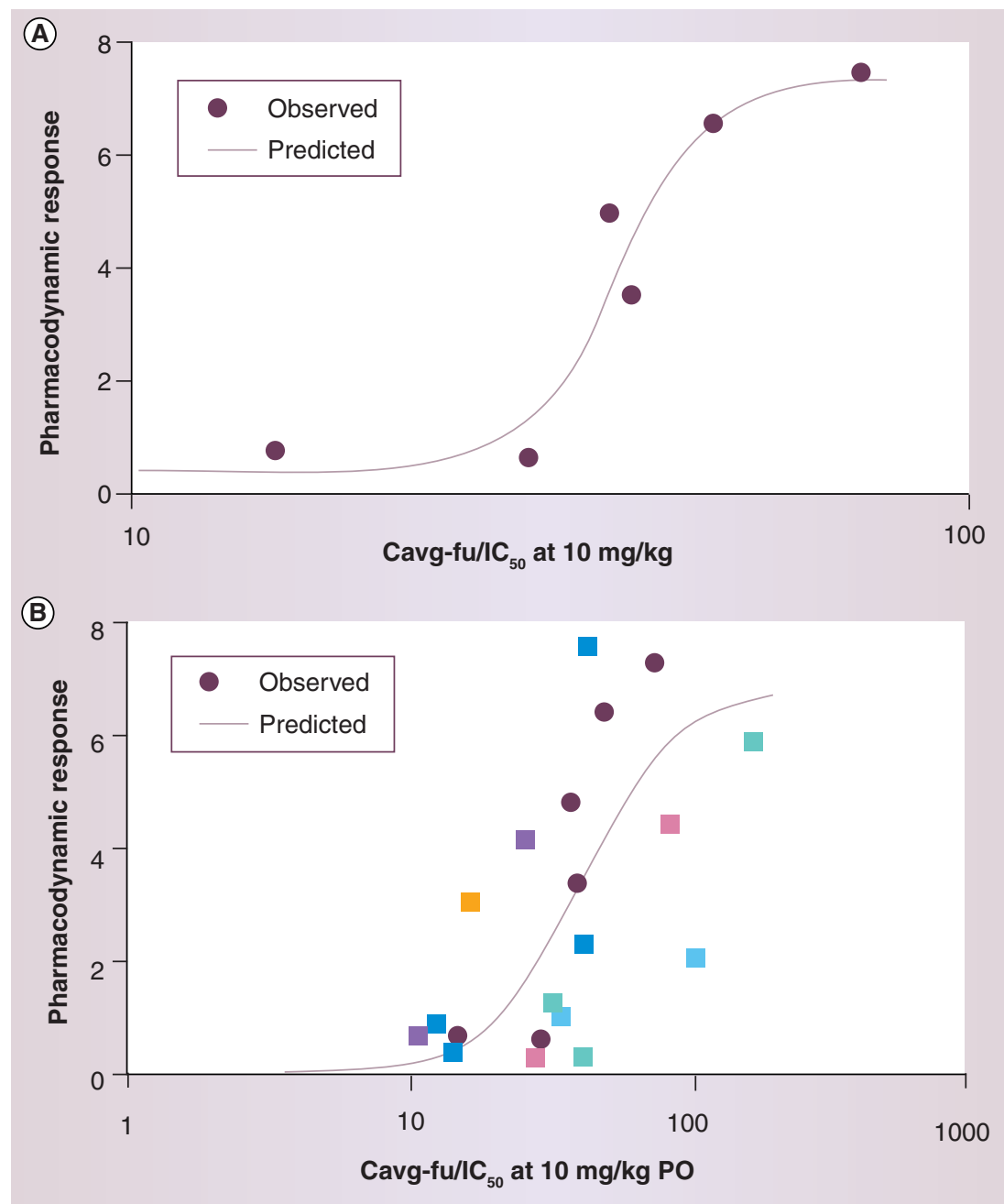

Figure 2. Exposure-pharmacodynamic response.

Cavg-fu: Averaged unbound plasma concentration following a single oral dose. in vivo pharmacology screening, resulting in a rapid identification of lead molecules. At level 2 , a time course for the PK/PD model is developed. The major pharmacodynamic parameters, including drug- and biological system-related parameters (e.g., PD response turnover rate, $\mathrm{K}_{\text {out }}$ ) are determined. At level 3, the established models are applied to the prediction of pharmacological response at different dosing regimens within a species and further to interspecies scaling. The biological system-related parameters usually vary between species, individuals and disease state [30]. Therefore, not only interspecies scaling, but also interpopulation/subject variability needs to be considered [30]. By contrast, the drugspecific parameters (e.g., target-binding affinity) are often comparable across species and thereby do not generally require interspecies scaling [30] At level 3, the model is being further validated and optimized as more in vivo pharmacology data becomes available.

\section{Integration of biomarkers \& systems biology into PK/PD modeling \& simulation}

A biomarker is defined as a characteristic that is objectively measured and evaluated as an indicator of normal biological processes, pathogenic processes or pharmacological responses to a therapeutic intervention [35,36]. Clinically validated or reliable biomarkers may sometimes be used as surrogates for clinical end points [35]. Biomarkers are particularly valuable for PK/PD model development in the drugdiscovery phase [37]. As previously mentioned, the development of mechanistic PK/PD models is typically based on the data of biomarker responses. Linking biomarkers with $\mathrm{PK} / \mathrm{PD}$ models gives us a better understanding of the mechanism of drug action, builds up confidence in the PK/PD model and provides the quantitative basis for translational research and for more accurate interspecies scaling [37]. This is especially important for unprecedented or novel targets, for which no PK/PD model exists. The application of biomarkers is also critical to some drug-discovery projects that have a low throughput of in vivo screening as a result of the requirement for longer term studies (i.e., obesity and osteoporosis). The use of integrated biomarker and PK/PD models for predicting biomarker response and, ultimately, clinical outcome, facilitates quantitative decision making on the selection of drug candidates, dosing regimens and study designs [38]. Finally, the 
addition of any new biomarker into PK/PD models should be applied with caution as some 'biomarkers' are simply, or coincidently, associated with pharmacological response and not always altered by therapeutic intervention [37].

Systems biology, an emerging discipline, uses an integrated approach to study and understand the function of biological systems and how the components of a complex biological system dynamically interact within the system [39]. It is likely to become increasingly important, as data linking specific drug target/pathways to clinical outcome become available. Systems biology has both experimental and computational components. The development of systems biology is an iterative process by which the experimental and computational components constantly inform each other. Systems biology not only sequentially integrates the data and knowledge of a drug target pathway, but also horizontally links the target with other related pathways. Linking the systems biology with a PK/PD model allows us to probe how a therapeutic intervention perturbs the system and is thereby capable of predicting not only on-target pharmacological effects, but also off-target effects [40].

\section{Future challenges \& directions for PK/PD modeling \& simulation}

As science (particularly the multi-omics and translational sciences) evolve, more and more biomarkers are being studied and identified, thereby facilitating drug discovery and development. This will add increasing complexity into PK/PD models and demand that the PK/PD scientists expand knowledge in many disciplines in order to appropriately integrate new data into PK/PD models [41]. As the model complexity increases, effective team work and collaboration are paramount, including collaboration between medicinal chemists, biologists, pharmacologists, preclinical scientists, clinicians, pharmacometrians, biostatistians and regulatory specialists. In turn, the communication within a project, or across projects, and particularly with the regulatory agency, may become another challenge to modelers [42].

Pharmacokinetic/PD modeling and simulation have evolved as a quantitative pharmacology and are an invaluable tool for drug discovery and development. It can streamline decisionmaking in compound selection, dose selection, study design, patient-population selection and product labeling. Ultimately, the use of PK/PD modeling and simulation should accelerate the delivery of better/safer treatments to patients. It will be an area of increasing importance in drug discovery and development in the future.

\section{Acknowledgements}

The authors thank Michelle Albright, Jay Mitchell and Wade Walk for editing the manuscript and Eillen Grover for collecting references.

\section{Financial \& competing interests disclosure}

The authors have no relevant affliations or financial involvement with any organization or entity with a financial interest in or financial conflict with the subject matter or materials discussed in the manuscript. This includes employment, consultancies, honoraria, stock ownership or options, expert testimony, grants or patents received or pending, or royalties.

No writing assistance was utilized in the production of this manuscript.

\section{Bibliography}

Papers of special note have been highlighted as:

- of interest

.. of considerable interest

1 van de Waterbeemd H, Gifford E. ADMET in silico modelling: towards prediction paradise? Nat. Rev. Drug Discov. 2(3), 192-204 (2003).

- Describes how in silico approaches will further increase our ability to predict and model the most relevant pharmacokinetic (PK), metabolic and toxicity end points and reviews different commercial software tools.

2 Ekins S, Waller CI, Swaan PW, Cruciani G, Wrighton SA, Wikel JH. Progress in predicting human ADME parameters in silico. J. Pharmacol. Toxicol. Methods 44(1), 251-272 (2000).
Wilson AGE, White AC, Mueller RA. Role of predictive metabolism and toxicity modeling in drug discovery - a summary of some recent advancements. Curr. Opin. Drug Discov. Devel. 6(1), 123-128 (2003).

4 Obach RS, Baxter JG, Wastall P. The prediction of human pharmacokinetic parameters from preclinical and in vitro metabolism data. J. Pharmacol. Exp. Ther. 283(1), 46-58 (1997).

- Describes several approaches by which human PK data can be predicted from preclinical data.

5 Ito K, Houston JB. Comparison of the use of the liver models for predicting drug clearance using in vitro kinetics data from hepatic microsomes and isolated hepatocytes. Pharm. Res. 21(5), 785-792 (2004).
6 Iwatsubo T, Hirota N, Ooie T et al. Prediction of in vivo drug metabolism in the human liver from in vitro metabolism data. Pharmacol. Ther. 73(2), 147-171 (1997).

7 Andersson TB, Bredberg E, Ericsson H, Sjoberg $H$. An evaluation of the in vitro metabolism data for prediction the clearance and drugdrug interaction potential of CYP2C9 substrates. Drug Metab. Dispos. 32(7), 715-721 (2004).

8 Sarawek S, Li L, Yu XQ et al. Examination of the utility of the high-throughput in vitro metabolic stability assay to estimate in vivo clearance in the mouse. Open Drug Metab. J. 3, 31-42 (2009).

- Investigates the utility of the high-throughput in vitro metabolic stability assay to generate an estimate of intrinsic clearance and to estimate in vivo clearance. 
9 Soars M, Webborn PJH, Riley RJ. Impact of hepatic uptake transporters on pharmacokinetics and drug-drug interactions: use of assays and models for decision making in the pharmaceutical industry. Mol. Pharm. 6(6), 1662-1677 (2009).

- Describes how metabolism-transporter interplay can influence hepatic metabolic clearance.

10 Mahmood I. Application of allometric principles for the prediction of pharmacokinetics in human and veterinary drug development. Adv. Drug Deliv. Rev. 59(11), 1177-1192 (2007).

11 Luttringer O, Theil FP, Poulin P, Schmitt-Hoffmann AH, Guentert TW, Lavé T. Physiologically based pharmacokinetic (PBPK) modeling of disposition of epiroprim in humans. J. Pharm. Sci. 92(10), 1990-2007 (2003).

12 Jones HM, Gardner IB, Waston KJ. Modeling and PBPK simulation in drug discovery. AAPS J. 11(1), 155-166 (2009).

13 Jones HM, Parrott N, Jorga K, Lave T. A novel strategy for physiologically based predictions of human pharmacokinetics. Clin. Pharmacokinet. 45(5), 511-542 (2006).

14 Davies B, Morris T. Physiological parameters in laboratory animals and humans. Pharm. Res. 10 (7), 1093-1095 (1993).

15 Brown RP, Delp MD, Lindstedt SL, Rhomberg LR, Beliles RP. Physiological parameter values for physiologically based pharmacokinetic models. Toxicol. Ind. Health 13(4),407-484 (1997).

16 Schmitt W, Willmann S. Physiology-based pharmacokinetic modeling: ready to be used. Drug Discov. Today Technol. 1(4), 449-456 (2004).

17 Fang L, Sun D. Predictive physiologically based pharmacokinetic model for antibodydirected enzyme prodrug therapy. Drug Metab. Dispos. 36(6), 1153-1165 (2008).

18 Kummar S, Kinders R, Rubinstein L et al. Compressing drug development timelines in oncology using phase ' 0 ' trials. Nat. Rev. Cancer 7(2), 131-139 (2007).

19 LoRusso PM. Phase 0 clinical trials: an answer to drug development stagnation? J. Clin. Oncol. 27(16), 2586-2588 (2009).

20 Marchetti S, Schellens JHM. The impact of FDA and EMEA guidelines on drug development in relation to Phase 0 trials. Br. J. Cancer 97(5), 577-581 (2007).

21 Sheiner LB, Steimer JL. Pharmacokinetic/ pharmacodynamic modeling in drug development. Annu. Rev. Pharmacol. Toxicol. 40, 67-95 (2000).
- Reviews the broad roles of modeling and simulation in drug development.

22 Uchizono JA, Lane JR. Emprircal pharmacokinetic/pharmacodynamic models. In: Pharmacometrics. Ette I, Williams PJ (Eds). John Wiley \& Son Inc., Hoboken, NJ, USA, 529-546 (2007).

23 Dokoumetzidis A, Iliadis A, Macheras P. Nonlinear dynamics in clinical pharmacology: the paradigm of cortisol secretion and suppression. Br. J. Clin. Pharmacol. 54(1), 21-29 (2002).

24 Rohatagi S, Carrothers TJ, Kuwabara-Wagg J, Khariton T. Is a thorough QTc study necessary? The role of modeling and simulation in evaluating QTc prolongation potential of drugs. J. Clin. Pharmacol. 49(11), 1284-1296 (2009).

25 Yao Z, Dubois DC, Almon RR, Jusko WJ. Pharmacokinetic/pharmacodynamic modeling of corticosterone suppression and lymphocytopenia by methylprednisolone in rats. J. Pharm. Sci. 97(7), 2820-2832 (2008).

26 Overgaard RV, Holford N, Rytved KA, Madsen H. PKPD model of interleukin-21 effects on thermoregulation in monkeysapplication and evaluation of stochastic differential equations. Pharm. Res. 24(2), 298-309 (2007).

27 Yamasaki Y, Tiran J, Albisser AM. Modeling glucose disposal in diabetic dogs fed mixed meals. Am. J. Physiol. 246(1 Pt 1), E52-E61 (1984).

28 Ashford JW, Schmitt FA. Modeling the time-course of Alzheimer dementia. Curr. Psychiatry Rep. 3(1), 20-28 (2001).

29 Simeoni M, Magni P, Cammia C et al. Predictive pharmacokinetic-pharmacodynamic modeling of tumor growth kinetics in xenograft models after administration of anticancer agents. Cancer Res. 64(3), 1094-1101 (2004).

- Describes a unique PK/pharmacodynamic (PD) model for discovery of anticancer agents.

30 Danhof M, de Jongh J, De Lange E, Pasqua OD, Ploeger BA, Voskuy RA. Mechanism-based pharmacokineticpharmacodynamic modeling: biophase distribution, receptor theory, and dynamical systems analysis. Annu. Rev. Pharmacol. Toxicol. 47, 357-400 (2007).

31 Yu XQ, Kramer J, Moran L et al. PK/PD modeling of 2-acetyl-4(5)-tetrahydroxybutyl imidazole-induced peripheral lymphocyte sequestration through increasing lymphoid S1P. Xenobiotica 40(5), 350-356 (2010).

-. Describes how to develop a mechanistic PK/PD model using a biomarker to connect PK and PD.
32 Beier H, Garrido MJ, Christoph T, Kasel D, Troconiz IF. Semi-mechanistisc pharmacokinetic/pharmacodynamic modelling of the anticociceptive response in the presence of competitive antagonism: the interaction between tramadol and its active metabolite on $\mu$-opioid agonism and monoamine reuptake inhibition, in the rat. Pharm. Res. 25(8), 1789-1797 (2008).

33 Gupta P, Friberg LE, Karlsson MO, Krishnaswami S, French J. A minimechanistic model of CP-690,550-induced reduction in neutrophil counts in patients with rhenmatoid arthritis. J. Clin. Pharmacol. (2009) (Epub ahead of print).

34 Jonsson EN, Macintyre F, James I, Krams M, Marshall S. Bridging the pharmacokinetics and pharmacodynamics of UK-279,276 across healthy volunteers and stroke patients using a mechanistically based model for targetmediated disposition. Pharm. Res. 22(8), 1236-1246 (2005)

35 Biomarkers Definitions Working Group. Biomarker and surrogate end points: preferred definitions and conceptual framework. Clin. Pharmacol. Ther. 69(3), 89-95 (2001).

- Describes the concept and application of biomarkers in drug discovery and development.

36 Frank R, Hargreaves R. Clinical biomarkers in drug discovery and development. Nat. Rev. Drug Discov. 2(7), 566-580 (2003).

37 Colburn WA. Biomarkers in drug discovery and development: from target identification through drug marketing. J. Clin. Pharmacol. 43(4), 329-341 (2003).

38 Krishna R, Herman G, Wagner JA. Accelerating drug development using biomarkers: a case study with Sitagliptin, a novel DPP4 inhibitor for Type 2 diabetes. AAPS J. 10(2), 401-409 (2008).

39 Davidov E, Holland J, Marple E, Naylor S. Advancing drug discovery through systems biology. Drug Discov. Today 8(4), 175-183 (2003).

40 Krishna R, Schaefer HG, Bjerrum O. Effective integration of systems biology, biomarkers, biosimulation, and modeling in streamlining drug development. J. Clin. Pharmacol. 47(6), 738-743 (2007).

41 Zhang L, Sinha V, Forgue T et al. Model-based drug development: the road to quantitative pharmacology. J. Pharmacokinet. Pharmacodyn. 33(3), 369-393 (2006).

42 Gobburu J, Marroum PJ. Utilisation of pharmacokinetic-pharmacodynamic modeling and simulation in regulatory decision-making. Clin. Pharmacokinet. 40(12), 883-892 (2001). 